\title{
A Magneto-Electric Dipole Antenna Array for Millimeter Wave Applications
}

\author{
Dang Thi Tu My \\ Quy Nhon University \\ Binh Dinh, Vietnam \\ dangthitumy@qnu.edu.vn \\ Tran Thi Huong \\ Huong Ban Mai Education Company Limited \\ Da Nang, Vietnam \\ huonggnouh@gmail.com
}

\author{
Huynh Nguyen Bao Phuong \\ Quy Nhon University \\ Binh Dinh, Vietnam \\ huynhnguyenbaophuong@qnu.edu.vn
}

\author{
Bui Thi Minh Tu \\ University of Da Nang, University of Science and \\ Technology \\ Da Nang, Vietnam \\ btmtu@dut.udn.vn
}

\begin{abstract}
In this paper, a low-profile broadband antenna is proposed for future 5G millimeter-wave cellular wireless networks. The proposed antenna is a modified Magneto-Electric (ME) dipole, which consists of four metallic plates, grounded vias, an aperture fed, a ground plane, and a microstrip line feed. The antennas are built on RT/Duroid 5880 substrates and have been realized by the printed circuit board technique. A singleelement with an overall of $10 \times 10 \times 1.04 \mathrm{~mm}^{3}\left(\sim 1.26 \lambda_{o} \times 1.26 \lambda_{o} \times 0.13 \lambda_{o}\right.$ at $38 \mathrm{GHz})$ exhibits an impedance matching of $27.9 \%(32.2-$ $42.8 \mathrm{GHz})$ for $\left|S_{11}\right|<-10 \mathrm{~dB}$ and a realized gain up to $7.5 \mathrm{dBi}$ over the frequency band. The usefulness of these antennas as beamforming radiators is demonstrated by a $1 \times 4$ element linear array. Also, a wide-band excitation is applied for the linear ME dipole array to realize a broadband array. The simulated results proved the proposed array can operate in a frequency band spreading from $31.4 \mathrm{GHz}$ to $42.1 \mathrm{GHz}$ with a gain of $12.5 \mathrm{dBi}$ and a side-lobe of $\mathbf{- 1 3 d B}$.
\end{abstract}

Keywords-magneto-electric dipole; millimeter wave; beamforming; antenna array

\section{INTRODUCTION}

The fourth generation (4G) Long Term Evolution (LTE) of cellular communication has been successfully implemented in many countries and areas. With a carrier frequency spectrum ranging from $700 \mathrm{MHz}$ to $2.6 \mathrm{GHz}$, today's cellular providers attempt to deliver high quality, low latency video and multimedia applications for wireless devices. However, the rapid increase of mobile data and the use of smartphones is creating unprecedented challenges to the wireless service providers. To address this, there has been a growing interest in cellular systems for the so-called millimeter-wave $(\mathrm{mmW})$ bands, between 30 and $300 \mathrm{GHz}$, where the available bandwidths are much wider [1]. Next generation of wireless cellular networks, which are known as fifth generation $(5 \mathrm{G})$, will make better use of the $\mathrm{mmW}$ bands $[2,3]$. Despite the absence of official standard for the $5 \mathrm{G}$, antennas for $5 \mathrm{G}$ cellular wireless networks have received much attention from the research community. It is well known that the path loss at the $\mathrm{mmW}$ bands is significant as compared to microwave bands due to the higher frequencies of mmW transmissions and the atmospheric absorption at the $\mathrm{mmW}$ frequency range. To mitigate the path loss at the $\mathrm{mmW}$ frequency range, beamforming has been proposed as an enabling technology for the $5 \mathrm{G}$ cellular wireless networks $[4,5]$. In general, an antenna for beamforming must be small, lightweight, low-cost, lowmutual-coupling in array environment, with pure polarization and high radiation efficiency, and easy to fabricate. On the other hand, magneto-electric (ME) dipole antennas, exhibiting wide impedance bandwidth and stable gain, have been presented for base stations in mobile communications [6, 7]. The basic design of the ME dipole consists of a magnetic dipole and an electric dipole, which are excited simultaneously to achieve a radiation having equal $\mathrm{E}$ - and $\mathrm{H}$-plane patterns and low back-radiation. Recently, the ME dipole antennas [8-11] have been scaled for $60 \mathrm{GHz} \mathrm{mmW}$ communications. The $\mathrm{mmW}$ ME dipole antennas are easily realized by using Printed Circuit Board (PCB) technology. However, these antennas were not characterized in an array environment. In addition, their magnetic dipoles are performed by two vertical quarterwavelength shorted patch antennas, thus, the antenna height is large.

This paper proposes a low-profile ME dipole antenna for use in $38 \mathrm{GHz}$ future $5 \mathrm{G}$ cellular wireless networks. The antenna is fed by an aperture slot, which ensures the excitation of the ME dipole antenna and achieves low-profile. Also, with wide-band excitations, the arrays yielded a wide bandwidth and side-lobe level better than $-13 \mathrm{~dB}$.

\section{SINGLE ELEMENT DESIGN}

This part presents the configuration of the proposed ME dipole antenna and analyzes its principles by investigating the current distribution. The optimization process is provided in detail to show the final design of the ME antenna. 


\section{A. Antenna Geometry}

Figure 1 shows the geometry of the antenna. The ME dipole antenna was designed on a substrate $\# 1$, which is a $W_{G N D} \times L_{G N D}$ RT/Duroid 5880 sheet with 2.2 relative permittivity 2.2, 0.0009 loss tangent, $0.7874 \mathrm{~mm}$ thickness, and $17 \mu \mathrm{m}$ copper thickness. The antenna consists of 4 metallic plates, 8 metallic posts, and a ground plane. The metallic posts with a diameter of $0.1 \mathrm{~mm}$ were realized by plated through-hole technology. The aperture slot was etched on the ground plane of the substrate \#1. A $50 \Omega$ microstrip line for feeding through the aperture was built on the substrate \#2 that is a RT/Duroid 5880 sheet with thickness of $0.254 \mathrm{~mm}$.

(a)

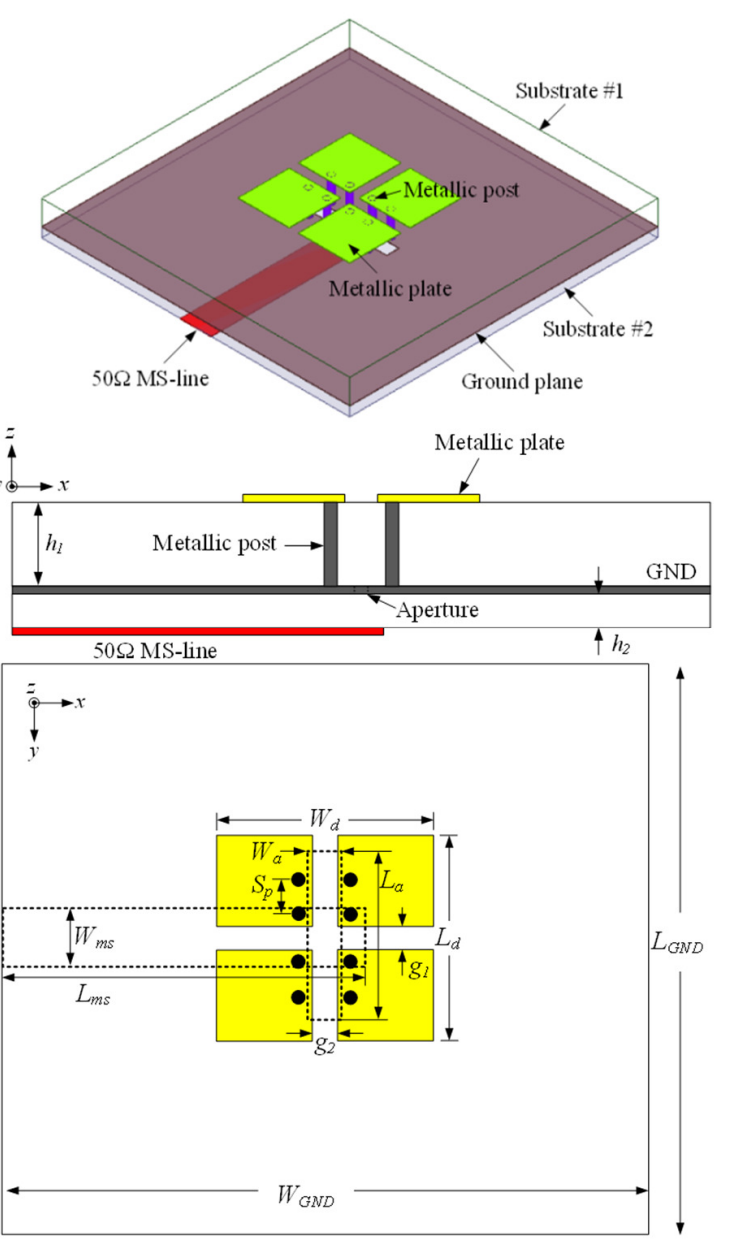

Fig. 1. Geometry of the proposed antenna: (a) perspective view, (b) crosssectional view, (c) top view.

\section{B. The ME Dipole Antenna Operation}

ANSYS Academic Research HF was used for all simulations of the proposed antenna. In order to realize an ME dipole antenna, the electric dipole and magnetic dipole should be excited simultaneously [4]. The metallic plates acted as the half-wavelength electric dipole antennas $\left(L_{d} \sim \lambda / 2\right)$. In general, the magnetic dipoles are performed by two vertical quarterwavelength shorted patch antenna [5-7]. In particular, due to the presence of the aperture slot fed, the magnetic dipoles are mainly performed by the slot edges between the shorted patch antennas. Therefore, the excitation of the ME dipole antenna can be obtained with a lower antenna height. This is demonstrated by the proposed antenna, which has a $0.7874 \mathrm{~mm}$ $\left(\sim 0.15 \lambda_{\text {eff }}\right.$ at $\left.38 \mathrm{GHz}\right)$ distance from the metallic plates to the ground plane. The ME dipole antenna operation can be seen in Figure 2, which shows the current distribution on the proposed antenna at $38 \mathrm{GHz}$ for different phase angles. At the phase angle of $0^{\circ}$, the currents on the slot edges between the shorted microstrip patches attain maximum strength, which means that the magnetic dipoles are excited. At the phase angle of $90^{\circ}$, the currents on the planar electric dipoles attain maximum strength at the surround edges, which means that the electric dipoles are excited. At the phase angle of $180^{\circ}$, the magnetic dipoles are excited again with opposite direction to the currents at the phase angle of $0^{\circ}$. At the phase angle of $270^{\circ}$, the electric dipoles are excited again with opposite direction to the currents at the phase angle of $90^{\circ}$. These indicate that the electric and magnetic dipoles are excited with $90^{\circ}$ phase difference consequently, the antenna obtains good broadside pattern and low back radiation [12].
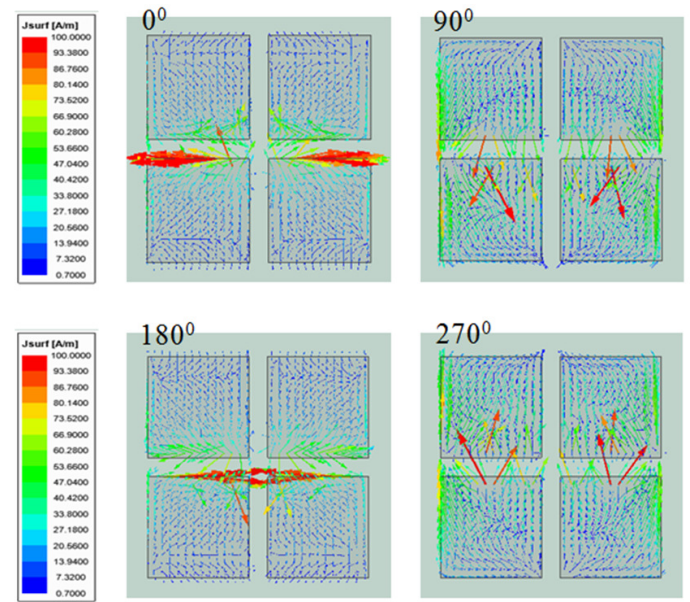

Fig. 2. Current distribution on the $\mathrm{ME}$ dipole antenna at $38 \mathrm{GHz}$ for different phase angles.

The resonant frequency of the proposed ME antenna can be optimized by changing its dimensions. In this case, the gaps between the metallic patches $\left(g_{1}\right.$ and $\left.g_{2}\right)$ are fixed at $0.3 \mathrm{~mm}$ while changing the length of ME dipole $L_{d}$. Figure 3 presents the S11 simulated with different values of $L_{d}$. As can be observed from this Figure, the resonant frequency of the ME antenna decreases while its length $L_{d}$ increases. The antenna shows a resonance at the desired frequency of $38 \mathrm{GHz}$ when the length of the antenna $\left(L_{d}\right)$ is equivalent to $3.46 \mathrm{~mm}$. Next, the input impedance of the antenna is verified to obtain the impedance matching. Due to the aperture fed, the ME dipole antenna's impedance mainly belongs to the length of the aperture slot while the width of the slot almost does not affect the input impedance of the proposed antenna, therefore, it was necessary to modify the length for getting a desired impedance matching. The length of the aperture slot $L_{a}$ is investigated when its width $W_{a}$ is fixed at $0.4 \mathrm{~mm}$. As can be seen in Figure 4 , the return loss of the antenna changed when the length $L_{a}$ 
changed. Furthermore, the resonant frequency of the antenna was hardly unchanged. This means that the input impedance of the ME antenna can be easily optimized for matching with the impedance of the microstrip line at the input. It is shown that the antenna yields a very good impedance matching as $L_{a}=2.05 \mathrm{~mm}$.

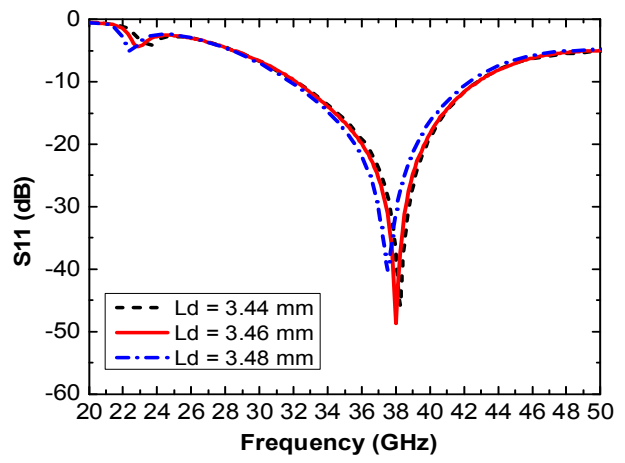

Fig. 3. S11 simulated results with different ME dipole length $L_{d}$.

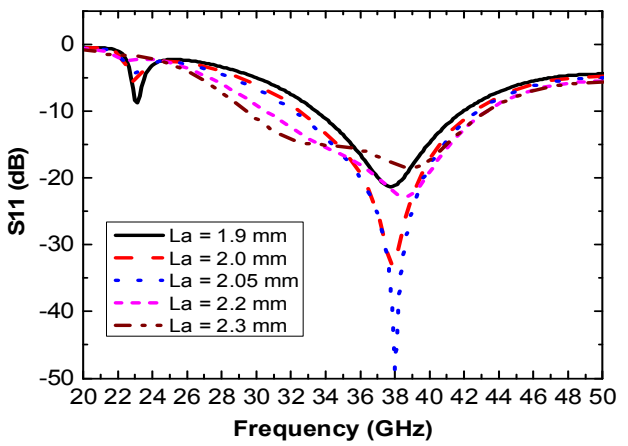

Fig. 4. Impedance matching investigation with different values of $L_{a}$.

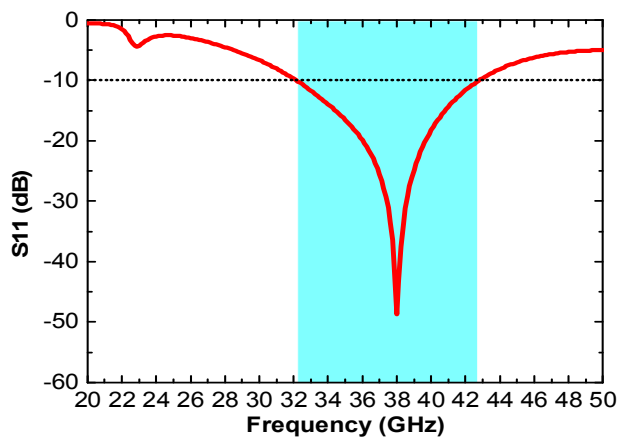

Fig. 5. Simulated S11 of the proposed ME antenna.

Figure 5 shows the optimized S11 of the ME antenna. It can be observed that the antenna has a wide bandwidth ranging from $32.2 \mathrm{GHz}$ to $42.8 \mathrm{GHz}$ with a center frequency of $38 \mathrm{GHz}$. The final design parameters of the antenna were: $W_{G N D}=10 \mathrm{~mm}$, $L_{G N D}=10 \mathrm{~mm}, \quad W_{d}=3.46 \mathrm{~mm}, \quad L_{d}=3.46 \mathrm{~mm}, \quad W_{m s}=0.733$, $L_{m s}=5.9 \mathrm{~mm}, W_{a}=0.4 \mathrm{~mm}, L_{a}=2.05 \mathrm{~mm}, g_{l}=0.3 \mathrm{~mm}, g_{2}=0.3 \mathrm{~mm}$, and $S_{p}=0.6 \mathrm{~mm}$. The radiation pattern simulated result of proposed ME antenna at $38 \mathrm{GHz}$ is given in Figure 6. It can be seen that the antenna yielded a directional radiation in $x z$ plane with a peak gain of $6.09 \mathrm{dBi}$ while posessing an omnidirectional radiation in the $x y$ plane.

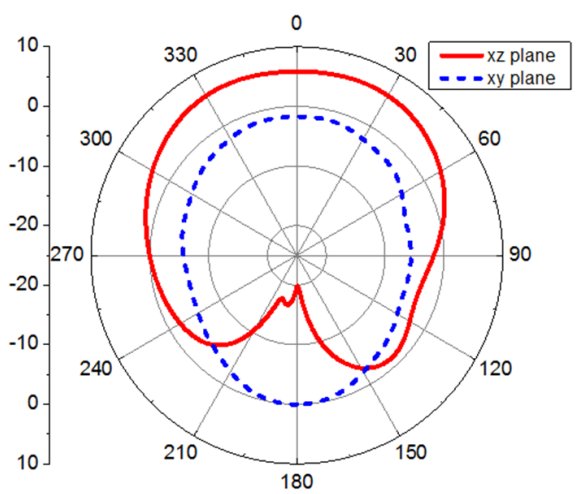

Fig. 6. Simulated radiation pattern of the proposed ME antenna at $38 \mathrm{GHz}$.

\section{ANTENNA ARRAY}

In this section, the ME antenna array design is divided into two parts. Firstly, the feeding network using T-junction power dividers is designed. The gradual transformer was used to realize a wide-band excitation. Then, 4 optimized ME antennas were integrated in a designed feeding network to form a 4element antenna array. Figure 7 presents the realized gain of the proposed ME antenna in its frequency band. It can be seen that the ME antenna has a realized gain up to $7.5 \mathrm{dBi}$ across the whole bands.

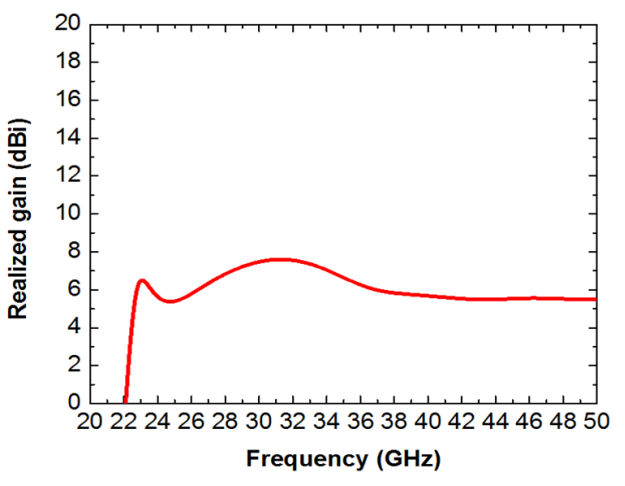

Fig. 7. Simulated realized gain of the single ME antenna.

\section{A. Feeding Network}

To realize the wide-band excitation, a feeding network composed of several equal $\mathrm{T}$-junction power dividers was implemented, as shown in Figure 8. The feeding network was built on an RT/Duroid 5880 sheet with a thickness of $0.254 \mathrm{~mm}$. Equal T-junction power dividers were implemented based on microstrip lines. To avoid the tiny width of the microstrip line with high characteristic impedance, each power divider contains a $50 \Omega$ to $25 \Omega$ transformer. The gradual transformer is inserted between the $50 \Omega$ and $25 \Omega$ microstrip lines to increase the impedance matching and reduce the losses at the corner of $25 \Omega$ lines. All corners of the divider are cut with an angle of $45^{\circ}$ in order to reduce reflection. Furthermore, the $\mathrm{V}$-shaped cuts are also etched at the middle points of $\mathrm{T}$ junction dividers for decreasing the reflected power. Figure 9 shows the simulated scattering parameters of the power divider. It can be seen that the divider shows a wide bandwidth spreading from $17 \mathrm{GHz}$ to $44 \mathrm{GHz}$ corresponding to all the 
transmission coefficients changing from 6.1 to $6.8 \mathrm{~dB}$. Thus, the proposed power divider is suitable for a wide-band array antenna design.

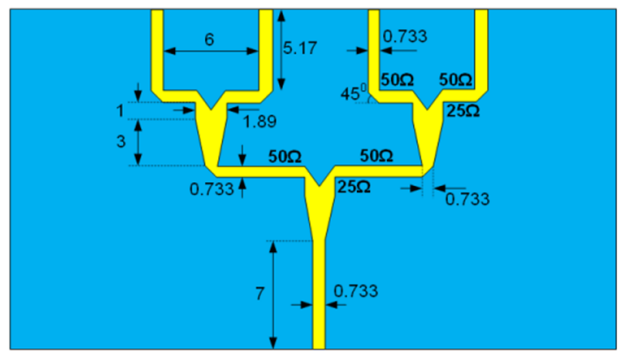

Fig. 8. Feeding network of wide-band excitation (all dimensions in $\mathrm{mm}$ ).

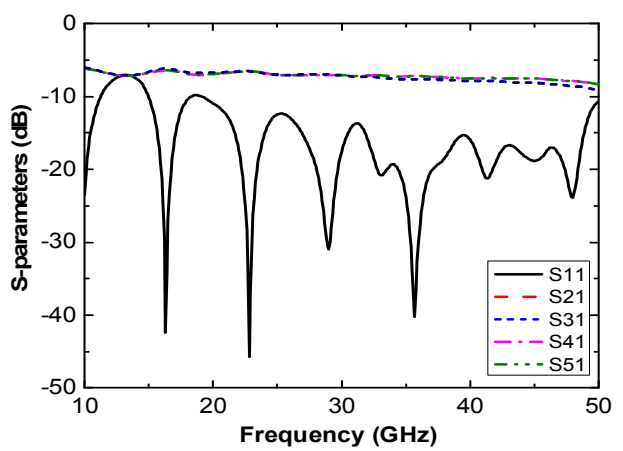

Fig. 9. Simulated S-parameters of the proposed wide-band excitation using $\mathrm{T}$-junction power dividers.

\section{B. Four Element Array Antenna}

In this work, four identical ME dipole antennas were integrated with the feeding network to create a higher gain antenna, as presented in Figure 10.

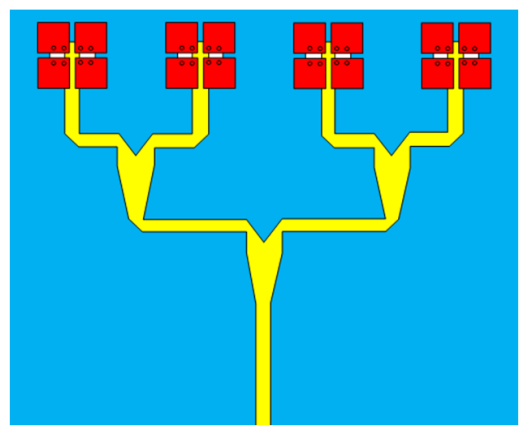

Fig. 10. Geometry of the proposed 4-elment array antenna.

The mutual couplings between two ME dipole antennas were studied for different values of center-to-center spacing. The final design was optimized with $6 \mathrm{~mm}$ spacing $(0.75 \lambda \mathrm{o}$ at $38 \mathrm{GHz}$ ). Besides, the length of the gradual transformer was also investigated for optimizing resonant frequency. For the best center frequency of the antenna, the length of gradual transformer was chosen as $2.8 \mathrm{~mm}$. The performance of the 4element array with equal-magnitude excitation at all elements is given in Figures 11-13. It can be observed from Figure 11 that the array can operate through the impedance bandwidth spreading from $31.4 \mathrm{GHz}$ to $42.1 \mathrm{GHz}$ with a center frequency of $38 \mathrm{GHz}$. Figure 12 shows the simulated results of the radiation patterns of the array at $38 \mathrm{GHz}$. As can be seen from this Figure, the array yielded a gain of $12.5 \mathrm{dBi}$ and a side-lobe of $-13 \mathrm{~dB}$ at $\mathrm{Phi}=90^{\circ}$, as shown in Figure 13.

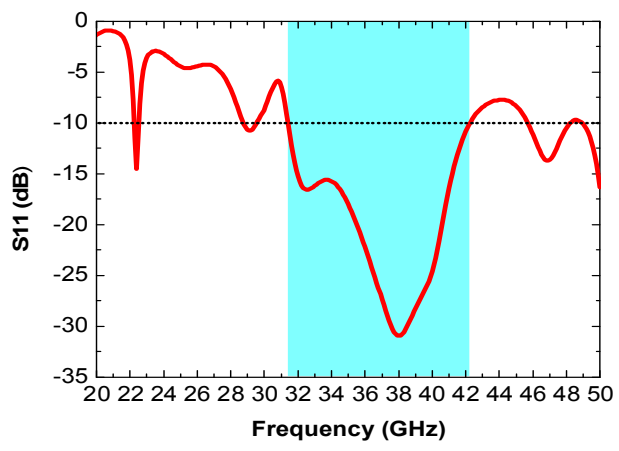

Fig. 11. Simulated S11 of the 4-element array.

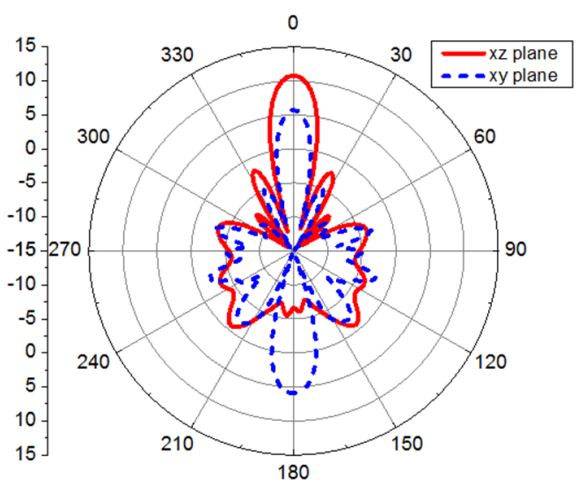

Fig. 12. Simulated radiation patterns of the 4-element array at $38 \mathrm{GHz}$.

The simulated result of the realized gain of the array over the frequency band is depicted in Figure 14. As can be seen in, the array antenna exhibits a stable realized gain from 10.8 to $12.5 \mathrm{dBi}$ in the operational frequency band. The comparison results between the proposed 4-element ME array antenna with the related works can be seen shown in Table I. The ME dipole antenna reported in [11] has a wider bandwidth as well as maximum realized gain compared to proposed ME dipole. However, the model in [11] has a complex structure since the mushroom-like EBG structures were used as a high impedance ground plane for increasing the bandwidth as well as the gain of the antenna. The $2 \times 2 \mathrm{ME}$ array proposed in [13] yielded a high directivity. But this antenna also has a complex structure by using a layer corporate-feed network based on Ridge Gap Waveguide.

TABLE I. COMPARISON BETWEEN THE PROPOSED AND REPORTED ME DIPOLE ANTENNAS

\begin{tabular}{|c|c|c|c|c|}
\hline Ref. & ME antenna & $\begin{array}{c}\text { Bandwidth } \\
(\mathbf{G H z})\end{array}$ & $\begin{array}{c}\text { Realized } \\
\text { gain (dBi) }\end{array}$ & Structure \\
\hline$[11]$ & Single & $23.4-41.5$ & 8.2 & Complex \\
\hline$[13]$ & Array $(2 \times 2)$ & $24-30$ & $15.4^{\mathrm{a}}$ & Complex \\
\hline \multirow{2}{*}{ Proposed } & Single & $32.2-42.8$ & 7.5 & Simple \\
\cline { 2 - 5 } & Array $(1 \times 4)$ & $31.4-41.2$ & 12.5 & Simple \\
\cline { 2 - 5 }
\end{tabular}




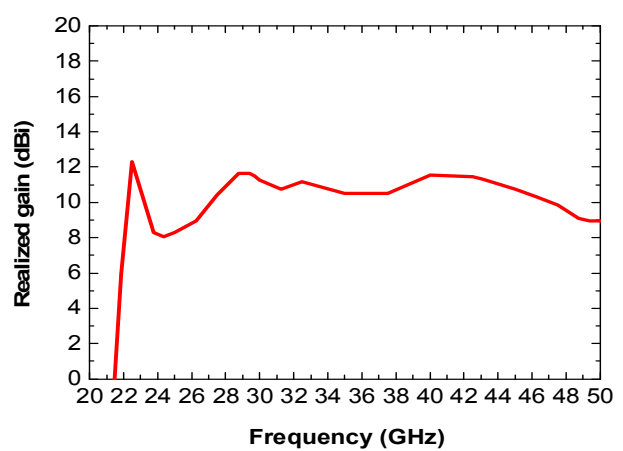

Fig. 13. Simulated broadside realized gain of the 4-element array.

\section{CONCLUSION}

A modified magneto-electric (ME) dipole antenna has been proposed for $\mathrm{mmW}$ use in this paper. The proposed antenna consists of four metallic plates which are connected to a ground plane via metallic posts. The antennas are fed by a microstrip line through an aperture slot to widen the bandwidth. The single-element shows a good impedance matching up to $27.9 \%$ and a maximum realized gain up to $7.5 \mathrm{dBi}$. A 4-element array has been designed for beamforming. The simulation results show that the array can operate in a frequency band ranging from $31.4 \mathrm{GHz}$ to $42.1 \mathrm{GHz}$ and yielded a gain of $12.5 \mathrm{dBi}$ with $-13 \mathrm{~dB}$-side lobe. The proposed ME antenna and its array can be a good candidate for applications in $\mathrm{mmW}$ systems.

\section{ACKNOWLEDGEMENT}

This work was conducted at the Calculation and Simulation Laboratory of the Faculty of Engineering and Technology, Quy Nhon University, Vietnam. The authors wish to thank the technical staff of the Laboratory for its support.

\section{REFERENCES}

[1] S. Rangan, T. S. Rappaport, and E. Erkip, "Millimeter-Wave Cellular Wireless Networks: Potentials and Challenges," Proceedings of the IEEE, vol. 102, no. 3, pp. 366-385, Mar. 2014, doi: 10.1109/JPROC.2014.2299397.

[2] H. Alsaif, "Extreme Wide Band MIMO Antenna System for Fifth Generation Wireless Systems," Engineering, Technology \& Applied Science Research, vol. 10, no. 2, pp. 5492-5495, Apr. 2020.

[3] M. Azhar and A. Shabbir, "5G Networks: Challenges and Techniques for Energy Efficiency," Engineering, Technology \& Applied Science Research, vol. 8, no. 2, pp. 2864-2868, Apr. 2018.

[4] W. Roh et al., "Millimeter-wave beamforming as an enabling technology for $5 \mathrm{G}$ cellular communications: theoretical feasibility and prototype results," IEEE Communications Magazine, vol. 52, no. 2, pp. 106-113, Feb. 2014, doi: 10.1109/MCOM.2014.6736750.

[5] O. M. Haraz, A. Elboushi, S. A. Alshebeili, and A.-R. Sebak, "Dense Dielectric Patch Array Antenna With Improved Radiation Characteristics Using EBG Ground Structure and Dielectric Superstrate for Future 5G Cellular Networks," IEEE Access, vol. 2, pp. 909-913, 2014, doi: 10.1109/ACCESS.2014.2352679.

[6] K.-M. Luk and B. Wu, "The Magnetoelectric Dipole-A Wideband Antenna for Base Stations in Mobile Communications," Proceedings of the IEEE, vol. 100, no. 7, pp. 2297-2307, Jul. 2012, doi: 10.1109/JPROC.2012.2187039

[7] L. Ge, X. Yang, Z. Dong, D. Zhang, and X. Zeng, "Reconfigurable Magneto-Electric Dipole Antennas for Base Stations in Modern Wireless Communication Systems," Wireless Communications and Mobile Computing, vol. 2018, May 2018, Art no. 2408923, doi: https://doi.org/10.1155/2018/2408923.
[8] K. B. Ng, H. Wong, K. K. So, C. H. Chan, and K. M. Luk, "60 GHz Plated Through Hole Printed Magneto-Electric Dipole Antenna," IEEE Transactions on Antennas and Propagation, vol. 60, no. 7, pp. 3129 3136, Jul. 2012, doi: 10.1109/TAP.2012.2196916.

[9] M. Li and K.-M. Luk, "A Wideband Circularly Polarized Antenna for Microwave and Millimeter-Wave Applications," IEEE Transactions on Antennas and Propagation, vol. 62, no. 4, pp. 1872-1879, Apr. 2014, doi: 10.1109/TAP.2014.2298246.

[10] M. Li and K.-M. Luk, "Wideband Magneto-Electric Dipole Antenna for 60-GHz Millimeter-Wave Communications," IEEE Transactions on Antennas and Propagation, vol. 63, no. 7, pp. 3276-3279, Jul. 2015, doi: 10.1109/TAP.2015.2425418.

[11] J. Sun and K.-M. Luk, "Wideband Magneto-Electric Dipole Antennas for Millimeter-Wave Applications with Microstrip Line Feed," in 2018 International Symposium on Antennas and Propagation (ISAP), Busan, South Korea, Oct. 2018.

[12] A. Chlavin, "A new antenna feed having equal E -and H-plane patterns," Transactions of the IRE Professional Group on Antennas and Propagation, vol. 2, no. 3, pp. 113-119, Jul. 1954, doi: 10.1109/TAP.1954.27983.

[13] W. Y. Yong, T. Emanuelsson, and A. A. Glazunov, "5G Wideband Magneto-Electric Dipole Antenna Fed by a Single-Layer CorporateFeed Network based on Ridge Gap Waveguide," presented at the 14th European Conference on Antennnas and Propagation, EuCAP 2020 Mar. 2020, Accessed: Jul. 05, 2020. [Online]. Available: https://research.utwente.nl/en/publications/5g-wideband-magnetoelectric-dipole-antenna-fed-by-a-single-layer.

\section{AUTHOR PROFILES}

Dang Thi Tu My received her B.Eng. and M.Eng. degrees from Hanoi University of Science and Technology in 2000 and 2004, respectively. From 2016 she is a Ph.D. student at the Department of Electronic and Telecommunication Engineering, University of Science and Technology, The University of Danang. From October 2000 to present, she is a lecturer at the Department of Technique and Technology, Quy Nhon University. Research interests: Electronics and Telecommunications, Ultra-high frequency and antenna technology, and artificial networks.

Huynh Nguyen Bao Phuong received the B.E, M.Sc. and D.E degrees from Hanoi University of Science and Technology, Vietnam in in 2003, 2007 and 2014, respectively. Now he is a senior lecturer of the Faculty of Engineering and Technology, Quy Nhon University, Vietnam. His research interests are high impedance surfaces (HIS), microstrip and metamaterial antennas, MIMO antennas.

Tran Thi Huong received the B.S degree in Electrical and Electronics Engineering from the Utsunomiya University, Japan in 2004 and M.E. and D.E degrees in Electrical and Electronics Engineering from Tokyo Institute of Technology, Japan in 2006 and 2010 respectively. From 2010 to 2019, a lecturer at the Department of Electronic and Telecommunication Engineering, The University of Da Nang, University of Science and Technology. Research interests: dielectric resonator, material measurements in $\mathrm{mmW}$ bands and high frequency planar antennas.

Bui Thi Minh Tu received her Ph.D. from the Universite de Technologie de Compiegne (France) in 2010. She started teaching at the Faculty of Electronic and Telecommunication Engineering, The University of Danang, University of Science and Technology in 2010. Since $01 / 2015$, she has been appointed as vice-dean of the Faculty. Her research interests include communication systems, signal processing, renewable energy, and sustainable development 